\title{
Planning Guide for a Total Market Approach to Increase Access to Family Planning-Module 1: Landscape Assessment
}

Christopher Brady

Laura Weeden

Jane Hutchings

Jerry Parks

Follow this and additional works at: https://knowledgecommons.popcouncil.org/departments_sbsr-rh How does access to this work benefit you? Let us know!

\section{Recommended Citation}

Brady, Christopher, Laura Weeden, Jane Hutchings, and Jerry Parks. 2016. "Planning Guide for a Total Market Approach to Increase Access to Family Planning-Module 1: Landscape Assessment." Seattle, WA: PATH and Washington, DC: The Population Council, Evidence Project. 


\section{The Evidence Project}

\section{Population Council}

4301 Connecticut Avenue, NW, Suite 280

Washington, DC 20008 USA

tel +12022379400

\section{evidenceproject.popcouncil.org}

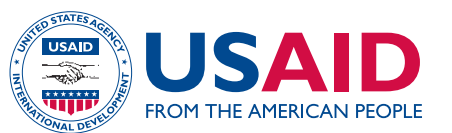

The Evidence Project is made possible by the generous support of the American people through the United States Agency for International Development

(USAID) under the terms of cooperative agreement AID-OAA-A-13-00087. The contents of this document are the sole responsibility of the Evidence Project and Population Council and do not necessarily reflect the views of USAID or the United States government.

The Evidence Project uses implementation science-the strategic generation, translation, and use of evidence-to strengthen and scale up family planning and reproductive health programs to reduce unintended pregnancies worldwide. The Evidence Project is led by the Population Council in partnership with INDEPTH Network, International Planned Parenthood Federation, Management Sciences for Health, PATH, Population Reference Bureau, and a University Research Network.

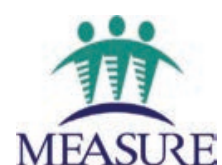

Evaluation
MEASURE Evaluation is funded by the United States Agency for International Development (USAID) and is implemented by the Carolina Population Center at the University of North Carolina at Chapel Hill, with Palladium Group (formerly Futures Group), ICF International, John Snow, Inc., Management Sciences for Health, and Tulane University.

PATH is driven by an unshakeable commitment to health equity and a bold belief in the power of innovation to improve health and save lives. For nearly 40 years, PATH has been a pioneer in translating bold ideas into breakthrough health solutions, with a focus on child survival, maternal and reproductive health, and infectious diseases.

For more information about total market approaches, contact:

PATH

Mailing address: PO Box 900922 Seattle, WA 98109 USA

Street address: 2201 Westlake Avenue, Suite 200 Seattle, WA, USA

Telephone: +1.206 .285 .3500$

Fax: +1.206.285.6619

Published in January 2016

Suggested Citation: Brady C, Weeden L, Hutchings J., Parks, J. 2016. "Planning Guide for a Total Market Approach to Increase Access to Family Planning. Module 1: Landscape Assessment.” Seattle, WA: PATH and Washington, DC: The Population Council, Evidence Project.

Cover photo: @ 2012 Akintunde Akinleye/NURHI, Courtesy of Photosphere.

Copyright (C) 2016 PATH. All rights reserved. 


\section{Planning Guide for a Total Market Approach to Increase Access to Family Planning}

Module 1: Landscape Assessment

CHRISTOPHER BRADY, SENIOR PROGRAM OFFICER, MARKET DYNAMICS, PATH LAURA WEDEEN, SENIOR POLICY AND ACCESS ADVISOR, PATH JANE HUTCHINGS, PROGRAM LEADER, REPRODUCTIVE HEALTH, PATH JERRY PARKS, PROGRAM ASSOCIATE, PATH 


\section{ACKNOWLEDGMENTS}

This document reflects the efforts of many individuals and organizations. USAID's Evidence Project has been the primary contributor of both financial and technical support and has played an essential role in the production of this document. PATH's total market approach work in the Democratic Republic of Congo, Myanmar, Nicaragua, and Vietnam were funded by the David and Lucile Packard Foundation and the Fred H. Bixby Foundation, which served as an important foundation for this guide. An ad hoc technical advisory group was formed to review the content and provide input. We appreciate the contributions of the following individuals and organizations in the development of this document:

Dominique Meekers, Tulane University, MEASURE Evaluation project

Jeff Barnes, Abt Associates

Kim Longfield, Population Services International

Ian Askew, Population Council

Anita Deshpande, Reproductive Health Supplies Coalition

Ben Light, United Nations Population Fund

Anna Mackay, Marie Stopes International

Mina Barling, Marie Stopes International

Maggie Farrell, US Agency for International Development

Ellen Weiss, Population Reference Bureau, Evidence Project

Kenneth Mugumya, Country Coordinator of the Uganda Family Planning Consortium 


\section{CONTENTS}

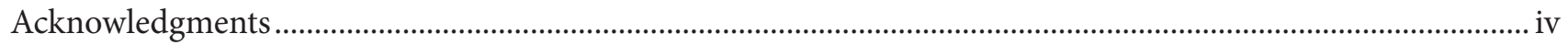

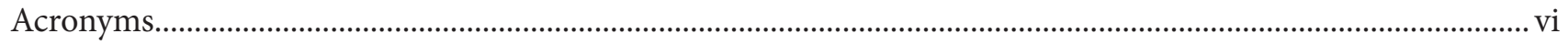

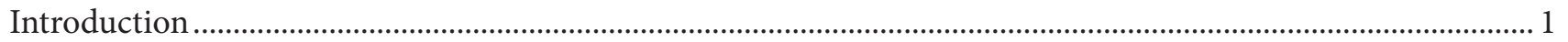

What is a total market approach to family planning? ...................................................................................... 1

When a total market approach to family planning may be useful ........................................................................ 2

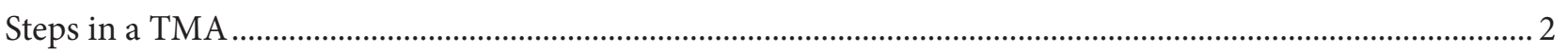

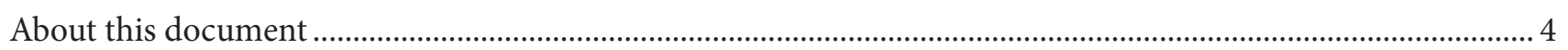

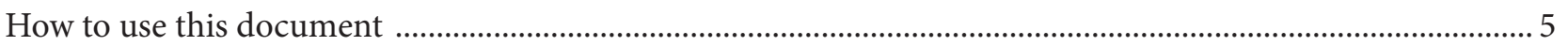

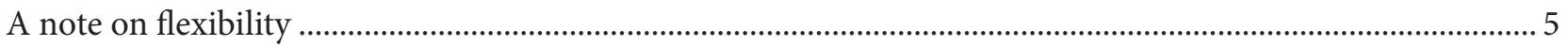

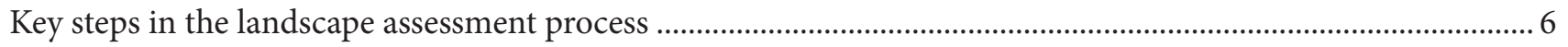

Step 1. Assess and catalyze interest ......................................................................................................................

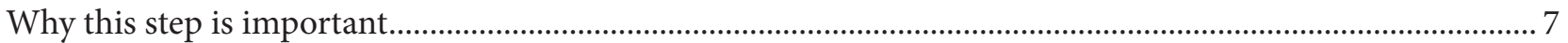

Background information for TMA planners ........................................................................................................ 7

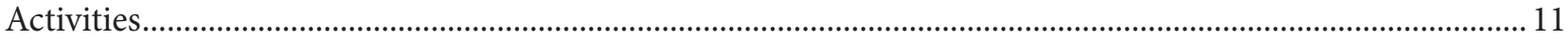

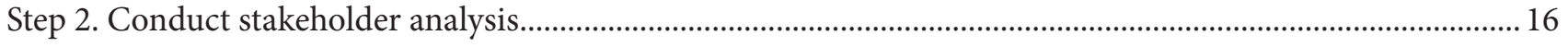

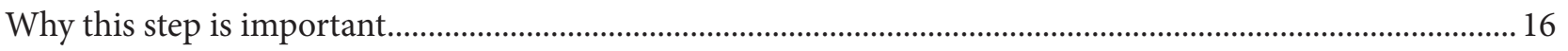

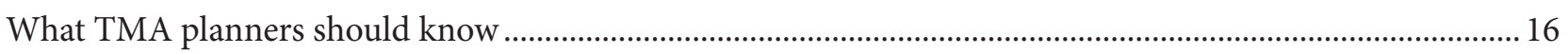

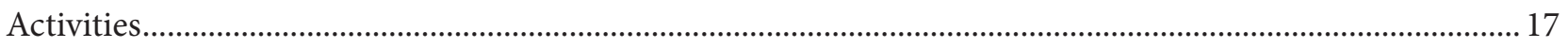

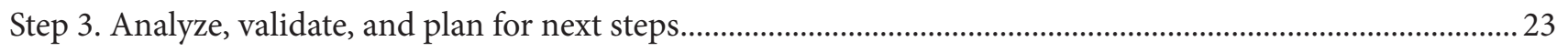

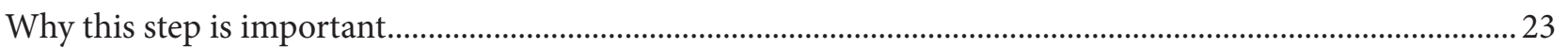

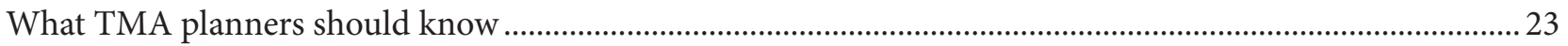

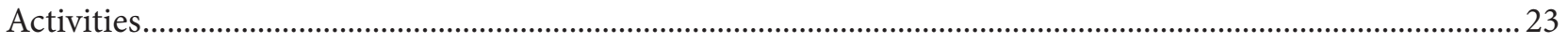

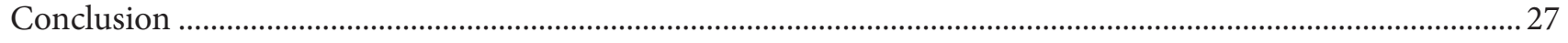

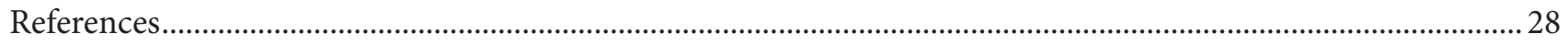

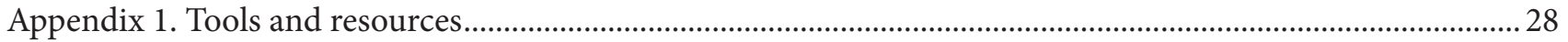




\section{ACRONYMS}

$\begin{array}{ll}\text { CPR } & \text { contraceptive prevalence rate } \\ \text { FP } & \text { family planning } \\ \text { IRB } & \text { institutional review board } \\ \text { LMICs } & \text { low- and middle-income countries } \\ \text { NGO } & \text { nongovernmental organization } \\ \text { RHSC } & \text { Reproductive Health Supplies Coalition } \\ \text { SWOT } & \text { strengths, weaknesses, opportunities, and threats TMA total market approach } \\ \text { UNFPA } & \text { United Nations Population Fund } \\ \text { USAID } & \text { US Agency for International Development }\end{array}$




\section{INTRODUCTION}

Worldwide, there is growing momentum and political will to ensure sustainable development and universal access to health products, care, and services. As part of this trend, governments are making a clear commitment to expand access to family planning (FP) services and products and are working to increase demand for a full range of contraceptive methods. Fundamental to this effort are questions about equity of access to FP, efficiencies in resource distribution, consumer preferences and behaviors, and costs.

This commitment to expand access comes at a time when international donor resources for FP are shrinking relative to current and expected demand. In particular, bilateral donors with a history of donating contraceptive commodities and national governments in low- and middle-income countries (LMICs) are increasingly embracing greater local responsibility for the financial, technical, and management aspects of FP programs. These developments are prompting governments to increasingly focus public-sector attention and scarce budgetary resources on segments of their populations with the least access to FP products and services.

These financial resource constraints, often exacerbated by rapidly growing populations, exist against a backdrop of added pressures for governments to help ensure universal health coverage.

\section{WHAT IS A TOTAL MARKET APPROACH TO FAMILY PLANNING?}

A total market approach (TMA) to FP is based on an understanding that meeting the diverse needs of various population segments equitably will require increased attention on coordination across sectors. It emphasizes the need for the public sector, nongovernmental organization (NGO) sector, and commercial sector to collaborate to increase access to FP for all segments of the population. Within this context, there is growing appreciation for the value of strategic coordination across sectors. In low-income countries, where donor funding continues to support a significant share of the health care system, a TMA can play an important role in building sustainable public-private partnership models. Many countries face acute or impending FP budgetary shortfalls, especially countries that are "graduating" from development assistance.

At its core, a TMA is useful for providing a framework and relevant data that can help planners facilitate a purposeful and constructive working relationship among the public, NGO (including social marketing organizations), and commercial sectors. These sectors often work from different information sources and may not be aware of one another's activities and focus. Because knowledge gaps are likely to persist, it is crucial for planners to align data across sectors.

For various reasons, completing all of the planned steps of a TMA described below may not always be possible. Therefore, depending upon the country situation (including available resources) FP planners may opt to apply specific TMA elements as entry points and lay the foundation for future work. In many cases, selecting entry points that are considered a high priority and that are less costly to address may be a more realistic option.
"A TMA leverages the comparative advantages

of all market sectors

to enhance equitable

and sustainable family

planning across market

segments with government

coordination and

support. It is based on

an understanding that

meeting the diverse needs

of different population

segments equitably

requires increased

attention on coordinating

across sectors." 


\title{
WHEN A TOTAL MARKET APPROACH MAY BE USEFUL
}

\author{
Certain factors and circumstances may help planners determine when a TMA is most useful. ${ }^{1}$
}

A TMA can be useful to help expand overall FP coverage and bring the private sector into government planning early, accelerating progress toward healthy, balanced markets. In particular, it helps focus subsidies toward those who need them the most, while directing private-sector services toward people who are willing and able to pay for services and products, either out of pocket or through other financing mechanisms. For example, a TMA may be appropriate in countries with one or more of the following circumstances, although there are many other situations where a TMA can play a constructive role:

1. Donors or governments are phasing out financial or commodity donations and technical assistance for FP programs. In the course of this shift, the public sector recognizes that it does not have sufficient resources to meet current and unmet demand, particularly serving the most vulnerable segments of the population. A TMA can complement greater domestic resource mobilization through increased revenue and tax collection with increased allocations for health care coming from the Ministry of Finance.

2. A range of donor-supported suppliers such as NGOs or social marketing programs using donor or public-sector resources are active in the contraceptive market. There is little coordination of investments or activities, resulting in these subsidized suppliers undercutting and displacing commercial suppliers or each other in the market. A misalignment of resources exists and long-term sustainability is jeopardized as consumers willing and able to pay may be taking advantage of subsidies.

3. Unmet need and especially unmet demand for family planning are poorly understood. More formative information and research on barriers that hinder increased demand and use within different populations and market segments is needed. This can take the form of geographic, behavioral, psychographic, and demographic segmentation of different consumer groups.

\section{STEPS IN A TMA}

As illustrated in Figure 1, the first phase in developing a national TMA plan is a landscape assessment. Key findings from this assessment and subsequent discussions with key stakeholders lead to the second phase, whereby a more in-depth and comprehensive assembly and analysis of FP market data takes place. This happens in conjunction with strengthening existing coordination mechanisms as well as engaging in targeted advocacy activities. This work is essential preparation for the third phase, the development of a comprehensive national TMA and monitoring and evaluation plan, which is followed by its implementation and ongoing monitoring work (the fourth phase).

This landscape assessment module was intended as the first of four planned modules addressing the steps needed to plan and implement a TMA. Modules 1 and 2 were developed jointly by PATH under the Evidence Project and the University of North Carolina/Tulane under the MEASURE project. As of publication of this document, the development and addition of the remaining two modules are pending. However, it is still useful to showcase the complete TMA process as part of this landscaping document. The following provides an overview of the main topics and suggested content covered in each of the four modules.

1 The likelihood and significance of these circumstances depend on how developed the market is for reproductive health products and services in a given country context. 


\section{Ongoing actions: TMA advocacy and stakeholder engagement}

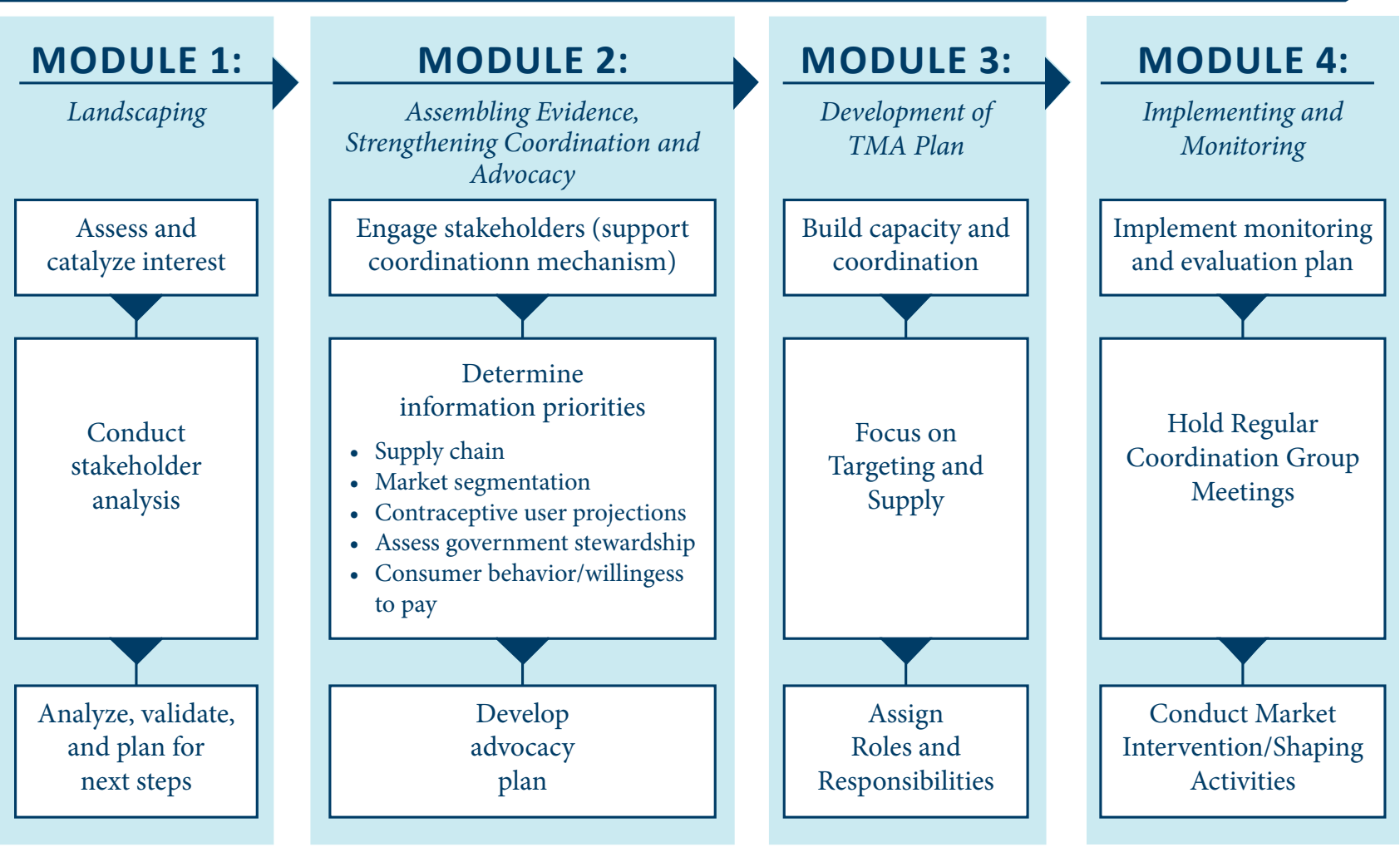

Figure 1. Planning a total market approach for family planning.

\section{Module 1. Landscape assessment}

- Assess and catalyze interest by conducting a desk review along with preliminary scoping among key stakeholders.

- Conduct stakeholder analysis.

- Analyze, validate, and plan for next steps.

\section{Module 2. In-depth analysis of the family planning market}

- Construct indicators, data requirements, measurement issues, and challenges.

- Identify data sources for secondary and primary research.

- Analyze patterns and trends in key TMA indicators. Identify key variables for stratification and market segmentation, how to measure wealth, analytical approach for TMA market analyses, estimate the number of FP users based on survey data.

- Develop dissemination and advocacy approaches.

- Assess government stewardship capacity. 


\section{Module 3. Development of TMA plan for FP}

- Convene coordinating group with government sponsorship.

- Forecast and establish TMA metrics.

- Assign roles and responsibilities for different TMA actors.

- Design results monitoring plan.

- Develop a budget and secure funding commitments.

- Establish consensus or workable agreement among key stakeholders and ensure requisite approvals within the government.

\section{Module 4. Implementation and monitoring}

- Conduct market intervention/shaping activities.

- Hold regular coordination group meetings and adjust plan to optimize strategies.

- Implement ongoing monitoring and evaluation component.

\section{ABOUT THIS DOCUMENT}

This document contains practical information and specific tools to help organizations and other in-country actors conduct a landscape assessment, the first phase in planning a TMA. The main body of this document is intended to be used in tandem with the TMA landscape assessment tools and resources in Appendix 1. Module 2 refers to market segmentation analysis strategies, and the future modules 3 and 4 will focus on developing a TMA plan and monitoring its implementation.

This module is likely to be most useful to organizations and individuals looking to plan a TMA, and we have designed it for a variety of experience and interest levels. Because a well-executed TMA benefits both the public and private sectors, representatives from any sector may initiate the process. For the purposes of this document, this group is collectively referred to as TMA planners.

TMA planners, the primary users of this module, include TMA practitioners, program managers, and other organizational representatives or consultants providing technical assistance in the FP arena. Secondary users include decision-makers at the country level involved in commodity security issues and supporting the growth of the commercial sector as part of an overall approach to commodity security (e.g., government officials, contraceptive security committees, local donors, and policymakers).

PATH, an international nonprofit organization that transforms global health through innovation, has a long history of public-private partnerships, including TMA for family planning, and has developed this resource in response to a need expressed by our partners for a simple document to share insights, experiences, and lessons learned across a range of organizations working to expand FP access and equity through markets. This module builds on general guidance provided in the Total Market Initiatives for Reproductive Health primer, ${ }^{1}$ the "Total Market Approach" course at the Global Health Learning Center, ${ }^{2}$ The SPARHCS Process Guide: A Planning Resource to Improve Reproductive Health Commodity Security, ${ }^{3}$ and the Using Total Market Approaches in Condom Programs primer. ${ }^{4}$ It also provides insights, useful practices, and lessons learned through the experiences of multiple organizations, particularly those associated with the Market Development Approach Working Group TMA workstream of the Reproductive Health Supplies Coalition (RHSC). 


\section{HOW TO USE THIS DOCUMENT}

This document includes:

- A description of each step in the landscape assessment process, information on why the step is necessary, what TMA planners should know, and a brief discussion of other key considerations.

- Key activities to achieve the goals of each step, along with recommended approaches and potential opportunities and challenges. Specific guidance on how to carry out each activity as well as insights gleaned from the experiences shared by FP practitioners who have conducted TMA-related work in a wide variety of country settings.

- Links to field-tested tools and other resources to help TMA planners perform specific activities.

\section{A NOTE ON FLEXIBILITY}

The framework presented in Figure 1 is intended to be descriptive rather than prescriptive. A TMA is best understood not as a step-by-step process but as an approach that provides clarity and structure to a health market. Market approaches are not intended to be standardized; their design and implementation vary depending upon the country environment and specific needs. Aspects of a TMA can also be incorporated into other country planning processes (e.g., upcoming FP planning processes such as the development of a national costed implementation plan).

There is no one best practice. Readers are urged to select and adapt information as needed. Different aspects of the methodology may be more or less relevant in different contexts and at different stages of market development. The amount of time required to conduct a landscape assessment can also vary significantly, depending on the availability of existing data, funding, and other contextual factors. In addition, steps within each module may overlap or occur in a different order depending on stakeholder priorities as well as available resources. Having the market data, which after analysis can indicate where the markets are seriously distorted, can help to convince actors of TMA's value and the need to collaborate more closely. However, to draw lessons from experience and measure success, TMA planning should be as systematic and deliberate as is feasible.

The authors realize that TMA planners often face budgetary constraints in terms of the overall scope for such an assessment. This guide was developed to be as comprehensive as possible, enabling TMA planners to pick and choose from different steps and methodologies as appropriate to the country needs and context.

A TMA is dynamic and evolving. This guide will also evolve over time to reflect changes in approaches, tools, experience, and circumstances. It is likely that sustained awareness building and advocacy will be needed to ensure that key stakeholders engage in the process of planning for a TMA. 


\section{KEY STEPS IN THE LANDSCAPE ASSESSMENT PROCESS}

A landscape assessment helps determine whether a TMA will contribute to a more equitable and sustainable FP environment in a given country and context. The objectives of the landscape assessment are to (1) understand the key issues affecting sustained and equitable access to and use of contraceptive products and services and (2) engage relevant stakeholders to identify the most effective levers of change in support of a TMA. Throughout the process, TMA planners need to be prepared to effectively describe what a TMA is and explain how it can support ongoing efforts to increase equity, access, and sustainability of FP programs. The amount of time and financial investment required to conduct this work depends on a variety of factors, such as whether the lead organization is based in country, availability of data, and other country-specific complexities encountered during the landscape assessment. The landscape assessment includes three steps (see text box).
MODULE 1

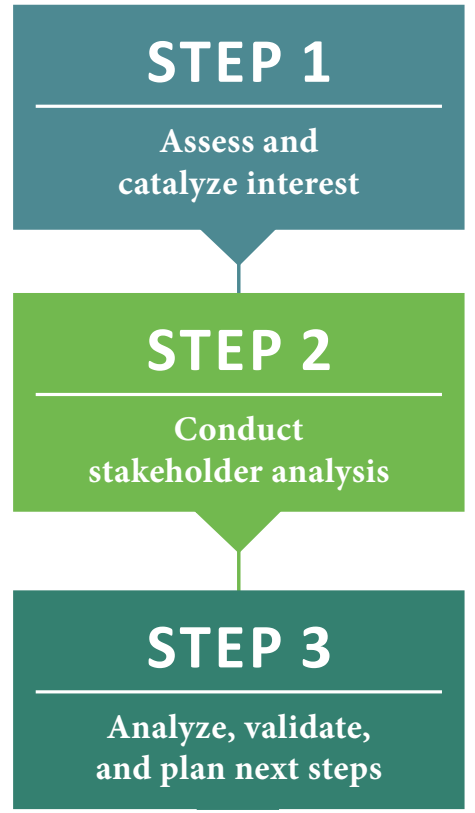

\section{THE THREE STEPS OF A TMA LANDSCAPE ASSESSMENT}

Step 1. Assess and catalyze interest. This step is an opportunity for TMA planners to begin stakeholder scoping with key informants to gauge stakeholder interests and concerns with regard to the FP market and to share information about the TMA and its value. It also allows TMA planners to examine existing data on market influencers, including supply-side issues, client demand, policy drivers and inhibitors, procurement, and other determinants of a sustainable and healthy FP market. At this stage, TMA planners interpret and use existing data to arrive at conclusions and compelling entry points that resonate with stakeholders. Nonetheless, it is recommended that in-depth data analysis be undertaken in Module 2. If findings from Step 1 suggest that a TMA would not be productive, planners have the opportunity to decide whether to pursue TMA programming further.

Step 2. Conduct stakeholder analysis. In this step, planners dig deeper by exploring key stakeholders' roles and interests while explaining how a TMA can contribute to meeting articulated national FP priorities and commitments. Through the stakeholder analysis process, planners can test and dig deeper into critical areas highlighted in Step 1 . Step 2 also provides robust insight into the perspectives of key organizations and individuals that influence markets.

Step 3. Analyze, validate, and plan next steps. Once planners have identified issues and concerns that a TMA may address, the next step is to conduct a light analysis of available data and bring stakeholders together to validate and prioritize the list of issues and concerns. Ultimately, the objective is to obtain stakeholder buy-in and chart a course for future total market programming. 


\section{STEP 1. ASSESS AND CATALYZE INTEREST}

\section{WHY THIS STEP IS IMPORTANT}

Conducting a landscape assessment of the FP market in a specific country or context requires conducting a desk review as well as soliciting the opinions of critical FP stakeholders active in the country. In Step 1, TMA planners assess and catalyze interest in a TMA, thereby setting the stage for a more rigorous stakeholder analysis in Step 2.

The idea that various products and services should have characteristics that need to be appealing to specific user groups is familiar to stakeholders in many contexts. Successfully implementing a TMA, however, takes a level of joint planning and coordination that is less common. In fact, FP programs in LMICs rarely make collective plans and decisions in the context of the total market. One possible outcome is that a TMA plan will be drafted and expressed in an FP strategy document (such as a costed implementation plan). It is critical that activities within the plan represent feasible actions as well as those that can be incorporated into existing work scopes. This involves having champions in place who appreciate the value of a TMA and can advocate for its use. It will then be up to the key market players to reference and use that information independently.

It is important, therefore, to begin the landscape assessment by understanding the FP environment and engaging a select group of stakeholders from three key sectors: public, NGO (including social marketing organizations), and commercial. Other important stakeholders that need to be involved are donors and multilateral organizations. Connecting with these groups early in the process allows TMA planners to highlight the benefits of working together toward healthier FP markets.

Because TMA programming is complex, resource intensive, and time-consuming, it requires deliberate planning and sustained commitment. Prior to a comprehensive landscape assessment, TMA planners are encouraged to hold initial discussions with relevant government stakeholders along with key FP donors supporting national efforts. This is an opportunity to review key TMA readiness considerations to identify what elements to focus on during the assessment work. There should be preliminary agreement among these stakeholders that there is sufficient interest, scope, and funding to justify the effort. It is important to emphasize that specific activities related to the use of fundamental TMA principles (engagement, advocacy, joint planning, dissemination, and monitoring) have dedicated costs that need to be acknowledged early on in the process. At the end of this step, TMA planners will be in a position to evaluate whether pursuing a TMA is warranted.

\section{BACKGROUND INFORMATION FOR TMA PLANNERS}

Prior experience with Step 1 suggests several useful considerations and themes.

\section{The role of the public sector is critical}

With support from international donors and technical agencies, the role of ministries of health in LMICs often focuses on provision of free or highly subsidized contraception to all segments of the population through national service delivery programs. Planning for a TMA requires the government to reassess its role in providing FP products and services. Depending on the circumstances, a TMA may require the government to gradually shift from acting as the primary provider 
of contraceptives to playing a broader role of coordinating or stewarding the social and commercial sector, and in many cases focusing its direct contribution-in terms of services and supplies-more sharply on reaching poorer and underserved communities.

TMAs are a part of health sector governance that can be best advanced through long term by government commitment and leadership given the public sector's responsibility for most statutory, regulatory and policy requirements As such, effective government stewardship of the TMA process and the ultimate results is essential for long-term success.

\section{The term total market approach is generally not well understood}

Stakeholders may not be familiar with the term total market approach, but they may relate to the concepts of health systems strengthening or public-private partnerships. Presenting a TMA in this context may be particularly useful when connecting government ministries and agencies other than the ministry of health, such as finance, planning, and drug regulatory agencies. In some cases stakeholders may be familiar with the term total market initiative, which is typically used when discussing specific, mixed health systems activities focused on a specific product or limited range of FP products..

\section{Development partners and other non-state actors can play a critical role in catalyzing interest}

It is generally not realistic to expect government agencies to act as the primary driver of TMA planning in the early stages. Often development partners and other non-state actors play a critical role in generating interest. These organizations and individuals can be considered "champions" who emerge during the stakeholder scoping with key informants exercise (Step 1, Activity 1.2), and who express an interest and willingness to drive the TMA process forward. If possible, it is important to identify TMA champions from each of the three sectors during the landscaping phase. One option includes looking at the extent to which the United Nations Population Fund (UNFPA) country office and a respected and capable NGO are willing and able to act as TMA champions. Identifying private-sector champions, such as representatives from pharmaceutical or other commercial firms, is also desirable at this early stage. Keep in mind, however, that the ultimate goal is for government to take increasing responsibility for driving the TMA forward, recognizing that the government may depend on technical and financial support from one or more of these champions. Key factors for evaluating potential TMA champions are capacity, workload, mandate, and relationships with other key actors in the contraceptive market. Their involvement is critical in terms of helping a government understand the TMA process, assess the utility of TMA in their country context, and make necessary plans to steward the initiative. There are many examples where NGOs, multilateral organizations, and donors have supported the secretariat of a multisectoral coordinating body with technical and financial assistance.

Depending on government priorities and capacity, ministries may choose to delegate elements of the TMA planning or implementation process to trusted development partners who are already well positioned to coordinate a diverse set of stakeholders. In every case, concerted efforts should be made to ensure government ownership-particularly as a TMA tends to involve a gradual shift away from subsidizing FP availability to the general public regardless of the ability to pay full cost.

\section{It is important to assess the government's capacity to carry out its stewardship responsibilities}

The government's role varies significantly from one context to another, depending on its willingness and ability to assume an active stewardship role. This stewardship does not require direct control of services and facilities; rather, stewards are responsible for engaging and orchestrating different partners to achieve common development goals. As stewards they 


\begin{tabular}{|c|c|c|c|}
\hline Responsibility & Policy and dialogue & Regulation & $\begin{array}{l}\text { Data collection } \\
\text { and analysis }\end{array}$ \\
\hline \multirow{6}{*}{ Components } & Mandate & Regulation & Data collection \\
\hline & $\begin{array}{l}\text { Sufficient funding } \\
\text { sources }\end{array}$ & $\begin{array}{l}\text { Sufficient funding } \\
\text { sources }\end{array}$ & Data analysis \\
\hline & Recognized need & Legal framework & Data management \\
\hline & Dialogue & & Data quality \\
\hline & $\begin{array}{l}\text { Monitoring and } \\
\text { evaluation }\end{array}$ & & Data dissemination \\
\hline & Human resources & & Data use \\
\hline
\end{tabular}

Table 1. Total market approach stewardship capacity framework.

Adapted from: Abt Associates. Total Market Approach Stewardship Capacity Assessment Report. Reproductive Health Supplies Coalition Innovation Grant V 1.0. [unpublished]. 2015.

should provide vision, guidance, facilitation, regulation, and evaluation measures to ensure that the various FP actors in the marketplace achieve results. Abt Associates has developed a TMA stewardship capacity framework (Table 1) to assess the capacity of governments to undertake specific stewardship functions. The tool was based on three major categories of stewardship capacity: (1) policy and dialogue, (2) regulation, and (3) data collection and analyses.

The objective of the stewardship capacity tool is to assess the government's ability to steward a total market approach by assessing its current ability to fulfill certain tasks and manage specific aspects of a multisectoral strategy. This tool generates scores for specific stewardship functions to guide future capacity-building activities related to stewardship. However, a minimum grade for a government entity to be deemed capable of stewarding a TMA has not been determined.

Conducting a cursory assessment of how the government meets these three stewardship tasks and responsibilities is an important part of the early landscape assessment process. Knowing the level of existing capacity in these areas provides a baseline and is a key criterion when developing a list of immediate priorities for TMA planning. However, as a TMA moves beyond the landscape assessment into more in-depth market analysis and planning, there is a need to assess stewardship areas with more precision. The results of this assessment will guide future plans, particularly in addressing areas of weakness.

Although a TMA may be compelling to governments in terms of meeting the expanding FP needs of their constituents, some public-sector actors may feel cautious. Government leaders may feel that they are competing with social marketing programs, or they may have concerns about distinguishing the public sector's core mission from that of the private (particularly commercial) sector. Governments that take a rights-based approach to health care provision may be less responsive to a TMA. This means explaining and providing examples of how a TMA can increase equity through reallocation of subsidies toward those in most need. 
Similarly, commercial-sector actors may not be in the practice of coordinating with each other or the government and may be resistant at first. They may see each other as competition or prefer to be under the radar to avoid undue scrutiny. Commercial-sector actors may also be reluctant to engage more fully with the government because of concerns about over-regulation or other policies unfavorable to their interests. Collaboration can also simply run contrary to the competitive environment that for-profit entities usually operate in. It is also worth noting that for many commercial suppliers, contraceptive markets represent a small share of their profits. This may influence decisions about whether it is prudent to devote senior staff time to discuss an unprofitable product line.

Active engagement with the commercial sector may not be a normative process; government partners may have varying levels of comfort and experience identifying this set of commercial partners and working with them. Nonetheless, familiarity and experience with public-private partnerships and health systems strengthening initiatives can be important foundations upon which to build total market programming. In more reluctant environments, advocacy takes longer and is likely to evolve out of existing multisectoral coordinatio mechanisms.

\section{PRIVATE-SECTOR BENEFITS OF TMA ENGAGEMENT}

There are many reasons why participating in a TMA will benefit the commercial sector:

- Commercial firms can get access to a variety of timely qualitative and quantitative market research reports.

- Better forecasting and more efforts to reach specific user groups reduces the likelihood of free and subsidized products crowding out commercial-sector brands as government and donor investments focus on reaching low-income groups.

- Commercial firms are in a position to influence policy and programmatic actions, which can free up market share for contraceptive products and services offered in the commercial sphere.

- Firms can engage in new business opportunities and partner with other organizations interested in growing the overall size of the contraceptive market. This could include taking advantage of opportunities to pool financial resources and to jointly develop non-brand-specific demand creation activities with other market actors.

- A TMA also provides the impetus and forum for commercial partners and social marketing entities to jointly develop strategies that seek to minimize direct competition. 


\section{ACTIVITIES}

\section{Activity 1.1. Desk review}

Conducting a desk review is important to understand the broader health and FP strategy and policy context when considering whether a TMA is likely to resonate with government and other parties' interests and concerns. A close look at national- and district-level policy documents as well as government strategies and commitments also offers insights into how the government perceives market shaping, which can help planners determine opportunities and potential obstacles. It also can highlight opportunities to align and leverage total market work from the outset and identify ways that the TMA links to and supports government-defined priorities. This information is critical later in the landscape assessment process to focus on key themes that resonate with leaders and demonstrate the value of a TMA as well as assess stakeholder networks.

This activity provides TMA planners with an in-depth understanding of available literature and other FP-related documents. Focus areas include key customers for FP; access to available products and services, including current pricing and charging practices; data on contraceptive usage; barriers to use; financing; and procurement of supplies. Through this process, TMA planners will have enough background to develop a preliminary list of key stakeholders to contact. Another goal at this stage is to highlight the most pressing topics for future discussions with government and a select number of other stakeholders.

At this stage, documentation and data for the desk review may be hard to find and collecting it may involve many steps. Important contextual data on the FP situation in a country may not be readily available on the Internet or through disseminated reports and publications. For example, content may be linked to gray literature such as reports, evaluations, and other documents that are less accessible through online and published records. Gathering these documents is likely to require deeper searches on organizations' websites and direct interaction with the most active FP stakeholders in a particular country.

Deeper investigation is required to find in-country resources, including the ministry of health reproductive health department or health statistics office, management information systems, and donor reports maintained by different organizations. Procurement and other useful market data can be found through the UNFPA Procurement Division in Copenhagen (e.g., the Reproductive Health Interchange), the RHSC (e.g., the Supplies Information Database), the International Planned Parenthood Federation, Population Services International, Marie Stopes International, and DKT International. Existing surveys such as the Demographic and Health Survey provide an overview of contraceptive use and its characteristics. When available, willingness-to-pay research and service-provision/private-sector assessments can supplement understanding of demand for FP products and services. A more comprehensive listing of reference materials can be found in Tool 1 (Appendix 1).

In some cases, the national drug authority may have data on specific (privately owned) products, and occasionally the commercial sector may be willing to share some sales data on their most popular brands, although usually commercial-sector stakeholders are reluctant to share this information. Market research

firms are another excellent source of commercial market data. For example, in many countries firms such as IMS Health and Nielsen track turnover of contraceptives through pharmaceutical outlets to estimate total market size for each product category. It should be noted that IMS Health is a commercial entity and usually charges a one-time access fee, which 
can be substantial. In many countries where a TMA may be considered, IMS data do not exist. This may be due to the small size of the commercial market or challenges involved in collecting relevant distribution data.

At this early stage, TMA planners are advised to avoid undertaking primary market research. Primary market research requires a significant investment of both time and resources, which should take place only after it has become clear that there is strong interest and commitment from government and other stakeholders in developing and implementing a TMA. As appropriate, this stage allows planners to identify key data gaps and build a strong advocacy case to pursue a TMA.

\section{KEY QUESTIONS FOR PRELIMINARY STAKEHOLDER INTERVIEWS}

- To what extent and in what ways will the idea of a total market intervention resonate with the government and other stakeholders?

- To what extent will there be opposition? Where and why?

- What is the best way to catalyze interest, capacity, and overcome resistance or skepticism?

- What is the best way to describe a TMA?

- What policy support exists for a TMA in FP and what policy barriers are there?

- Who are the most appropriate government partners to steward a TMA in FP?

- What are the levels of donor involvement in FP programming?

- Where does the financing and provenance of FP supplies come from?

- Is there scope and willingness to modify distribution mechanisms?

- Which NGOs and private commercial organizations are likely to be interested in collaborating and why?

- Do free supplies compete with subsidized and commercial markets?

- What is the estimated level of non-public engagement in the delivery of FP products and services?

- What coordination mechanisms already exist and are they appropriate for total market engagement moving forward? 


\section{Key questions to explore include:}

- How does country leadership see the health sector's role in its broader development agenda?

- What is the level of resources committed to strengthening health generally and family planning in particular?

- How does the government finance its national health strategies and plans, and to what extent is it reliant on external funds?

- Where are different consumer segments currently getting their FP products and services?

- What are the government's priorities and commitments in the FP sector?

- Is the government willing to redirect its efforts away from providing free contraceptive supplies to the urban middle class (focusing on the most vulnerable communities instead)?

- Do government strategies and plans recognize the contributions of the private sector?

- What mechanisms are in place to coordinate procurement and distribution efforts of different suppliers?

Tool 1 (Appendix 1) includes a full list of policy and FP questions to consider for the desk review.

Donor strategies in the health and FP domains are also an important component of the desk review. Donors and United Nations agencies can be strong advocates for public-sector stewardship, and a review of their country strategy documents can help TMA planners assess the extent and duration of external FP support..

\section{Tools for Activity 1.1}

Tool 1. Documents for general landscaping and desk review (key country development policy references and data sources related to family planning)

\section{ESSENTIAL TMA} RESOURCES

The resources listed below

will help TMA planners

identify and obtain useful

information:

- National health strategy

or plan.

- National health review documents.

- National reproductive health strategy or plan.

- National FP strategy or plan.

- FP 2020 costed

implementation plan.

- Country policies and plans related to decentralization, health financing, and universal health coverage.

- Donor strategies in the health and FP sectors related to FP.

- Strategic plan for the procurement of reproductive health commodity security.

- Annual reports for national reproductive health programs.

- Government organizational charts (organograms). 
Activity 1.2. Stakeholder scoping with key informants

The gathering and synthesizing of relevant FP and contraceptive market background documents creates a good foundation for a deeper, more nuanced total market perspective. It is now time to initiate contact with a small number of key informants to further explore relevant issues and questions and to test perceptions. At this point TMA planners should have identified government leaders and a select number of NGOs, professional health care associations, and commercial-sector stakeholders to meet with. The planners should also be in a position to identify appropriate local partners who can play a role as TMA champions. This smaller group of champions should be willing to support the TMA landscape assessment process and can assist in scheduling meetings with appropriate government partners and other in-country FP stakeholders if necessary.

When it comes to identifying key informants to speak with during this stakeholder scoping work, it is useful to remember that governments are not monolithic. Different government entities (e.g., departments of reproductive health or FP, government entities addressing universal health coverage within the ministry of health or state and regional governments, and ministries of finance) likely have different perspectives and insights on a TMA. Generally, it is most useful to first meet with the ministry or department that oversees the national FP program. To secure the meeting, it may be necessary to enlist the support of a development partner, such as a multisectoral group, donor, or an NGO that champions TMA.

The focus of these key informant meetings is to identify relationships and critical topics to explore more fully later during the stakeholder interviews described in Step 2. At this point it is useful to decide what elements are considered core requirements and can be easily evaluated when it comes to successfully pursuing a TMA agenda. Tools 2.1 and 2.2 (Appendix 1) provide useful background information to better understand where a specific country sits in terms of matching specific market development approaches as well as a list of country readiness considerations when evaluating total market-related work. These considerations highlight whether a TMA would be appropriate for a country along with suggested indicators to measure over time. They can also be used to filter the data gathered during interviews and focus on themes that were determined to be the most relevant during the landscape assessment.

Finding common language and references can be extremely helpful in framing successful conversations. In most cases, it is more manageable and sustainable to integrate total market interests into an existing multisectoral coordination mechanism, such as a contraceptive security committee or FP working group led by the ministry of health, rather than to create a separate mechanism. However, this must be specifically assessed in the landscape assessment and can be addressed during the stakeholder interviews (Activity 2.5).

As mentioned previously, it is important to understand what coordination mechanisms are potentially appropriate for the government to use to steward total market programming. It is also important to know whether these mechanisms are functional and the extent to which they include appropriate stakeholders. Contraceptive security committees or reproductive health technical working groups are natural forums to consider.

. Depending on the structure and expertise in a specific country, some TMA planners may have the opportunity to explore these questions with their own country-based staff, whereas others need to seek advice from external sources. Multilateral and bilateral donors or foundations that have provided significant support and/or have large programs operating in the country often have close working relationships with government leaders and are considered good resources. This is also a good opportunity to ask a few key informants for other relevant sources of data. 
Because complex hierarchies are common, it may be necessary to conduct multiple meetings with different government offices to introduce key ideas, explore needs, and achieve appropriate levels of buy-in and engagement. Key messages to communicate in these early scoping meetings include:

- Introduce the concept of a TMA. Outline what existing challenges can be addressed using a TMA, describing it as a practical, actionable approach to increase coordination with a focus on the comparative advantages of each sector to build an effective and sustainable market for FP. It is also an opportunity to share key data points gathered through the desk review (Activity 1.1).

- Acknowledge what the government has done so far related to a TMA in FP or other health areas that involve an emphasis on increased multisectoral coordination.

- Explain the importance of a TMA and link it to the attainment of national FP goals and objectives. It may also be useful to provide one or two practical examples of how this can be achieved. For example, a TMA supports efforts to meet the needs of individual user groups and can serve to improve the contraceptive prevalence rate equity differential between urban and rural communities. Engagement with multiple stakeholders and coordinated planning also are necessary in the implementation of national FP strategies that work toward a more effective and sustainable contraceptive market.

- Emphasize that TMA programming relies on a close alignment with government FP priorities and is designed to complement existing initiatives and plans.

- Underscore the importance of engaging with the private sector to increase access and improve efficiency.

- Discuss the importance of the government's stewardship role and how this facilitates and enhances FP outcomes.

- Explain the entire TMA process once the landscape assessment has been completed.

- Gain feedback from the government about key opportunities and challenges that might be expected as part of a national TMA plan..

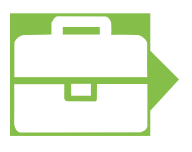

\section{Tools for Activity 1.2}

Tool 2: Considerations When Evaluating TMA-Related Work in Different Country Contexts.

Tool 3: Generic TMA Overview—Presentation 


\section{STEP 2. CONDUCT STAKEHOLDER ANALYSIS}

\section{WHY THIS STEP IS IMPORTANT}

TMA planners conducted a preliminary desk review in Step 1 and initiated contact with several key informants to decide whether to proceed with the next steps in the TMA process. Step 2 requires TMA planners to commit to a larger effort by conducting a stakeholder analysis, honing a list of stakeholders to interview, finding the best tools to gather information, and conducting interviews. This step also includes using the information collected from stakeholders to update the desk review and visiting FP providers and other outlets distributing contraceptives to collect consumer-level data.

Activities in Step 2 provide TMA planners with a more robust understanding of the FP market in the country, challenges or barriers that restrict its function, and opportunities to make necessary adjustments. Ideally, this work helps to clarify key questions and issues about the market itself, identify gaps in information that are essential to understanding the requirements of a total market, and explore the feasibility of potential solutions.

This deeper understanding should help to develop specific objectives and outcomes for the potential TMA based on local priorities that can support and inform existing strategies.

\section{WHAT TMA PLANNERS SHOULD KNOW}

As TMA planners think about which stakeholders to interview and what to ask them, it is useful to hone the objectives based on the desk review and initial discussions conducted in Step 1. Gathering insights from stakeholders is a complex process that depends on a variety of factors. Planners may find it useful to identify or map stakeholder networks and constituencies. As in Step 1, it is essential to identify stakeholders from the public sector, NGOs (including social marketing organizations), and commercial sector, and to focus on those who are the most active in the FP market as well as those

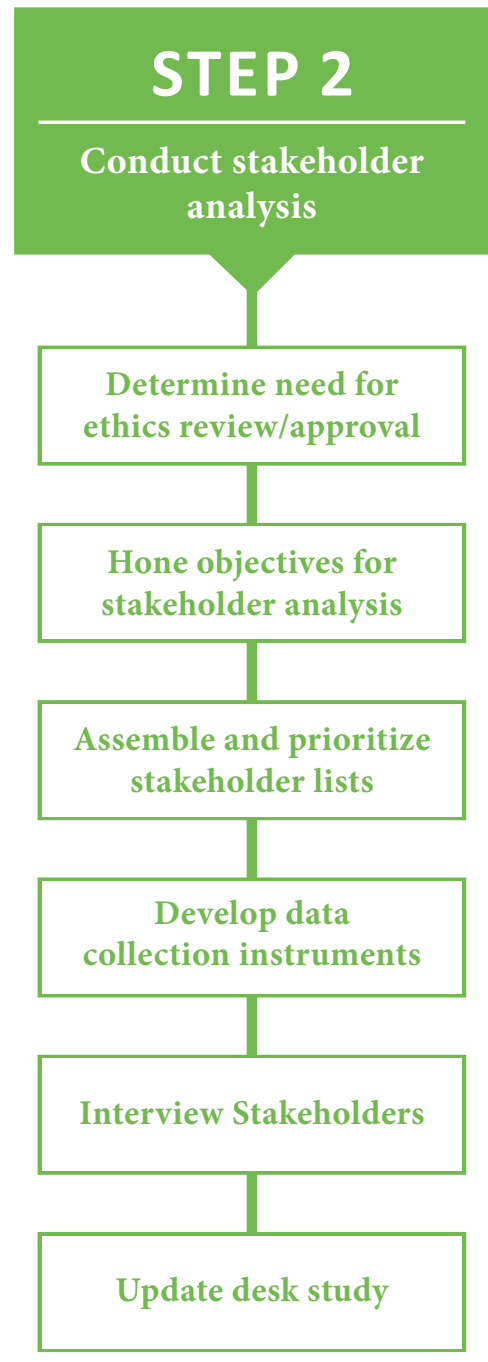

\section{WHAT IS STAKEHOLDER} ANALYSIS?

\section{WHAT DOES STAKEHOLDER} ANALYSIS ACHIEVE?
Stakeholder analysis is the process of

identifying the individuals or groups that are

likely to affect or be affected by a proposed

action. It also includes sorting them according

to their influence on the action and how a

specific action will affect them.

Stakeholder analysis can facilitate institutional

and policy reform processes, such as those

related to a total market, by accounting for

and incorporating the needs of those who

have a stake or interest in the reforms under

consideration. ${ }^{1}$ 
who may not be as readily visible, such as for-profit stakeholders. Tracking down meaningful commercial-sector data and identifying key stakeholders from that sector can be difficult. In most LMIC markets, justifying long-term investments for low-margin products and building a loyal consumer base can be challenging. In addition, commercial markets are often highly fragmented, lacking a unified direction, particularly when interacting with the government. Finally, key actors may be hesitant to share information that competitors could use to gain an edge and worry about government interference in their business. Tool 7 (Appendix 1) summarizes specific aspects of a TMA that are attractive and compelling to the commercial sector. Communicating these points is useful when engaging with the commercial-sector actors and explaining why being involved in a TMA is to their benefit.

\section{ACTIVITIES}

Activity 2.1. Determine need for research ethics review and approval for stakeholder analysis

Irrespective of the approach developed for the stakeholder analysis, an institutional review board (IRB) or research ethics committee review may be required to determine whether the stakeholder interviews are considered human subjects research and to ensure that all data collection activities meet the regulatory requirements for review of research protocols. A review may be necessary both within the organization carrying out the landscape assessment and at the country level. It may also be useful to find out whether conducting an informal market check (Activity 2.6) needs to be included in the protocol. As the process of interviewing stakeholders proceeds, informants may suggest other people to interview (snowball effect). This increases the chances of accumulating a robust list of individuals with different perspectives and insights.

The following tips can make this process smoother:

- Because an ethics review can take several weeks or longer, it is a good idea to begin as early as possible. It may also be possible to formally request a determination that the activity does not meet the definition used by the US government of research with human participants. This designation is particularly appropriate if one considers that, in the case of a total market assessment, the activity is intended to determine the feasibility and possible design of a future intervention, as opposed to collecting generalizable data for social science or clinical research purposes.

- If data are collected by an outside institution, an additional IRB review may be required by the home institution.

- IRBs require the study protocol, questionnaires, sampling procedures, and informed consent forms, and they request reassurances about data storage and certified translations of consent forms and questionnaires. Many IRBs also require documentation of human subjects' protection training.

- Finally, because some countries have multiple review boards with slightly different processes, it is useful to research which boards are relevant to this work and which are most efficient in their review processes.

- Many IRBs have resources available to assist in planning for and completing an IRB review and are happy to meet with teams to help them complete their submissions.

\section{Tools for Activity 2.1}

Tool 4: Guidance on the Research Determination Process, Sample Research Protocol on TMA Landscaping, Sample Informed Consent Form, and Sample Generic Stakeholder Interview Guide 


\section{Activity 2.2. Hone objectives for stakeholder analysis}

Stakeholder analysis is a critical step to gain insight on key priorities, potential bottlenecks, areas of concern, and feasibility of the approach. It also gives credibility to the assessment process and contributes to local ownership of the findings and recommendations of the assessment. On a practical level, stakeholder analysis provides an additional opportunity to close information gaps and supplement the literature review process, because some information is not accessible any other way.

Based on the desk review findings, TMA planners should clearly articulate the objectives for the stakeholder analysis. Interviews with stakeholders can have multiple and varied objectives; the more objectives being pursued, the more complex, expensive, and time-consuming the exercise will be. To the extent possible, therefore, it is valuable to prioritize specific areas of interest and focus on only the most critical pieces of information when querying the stakeholders. Potential objectives include:

- Understand FP stakeholder networks across sectors and the existence and nature of functional relationships between stakeholders.

- Identify specific issues and priorities in a country.

- Determine which of these issues and priorities are likely to lend themselves to total market solutions.

- Understand FP policies and systems that would be involved in implementation of a TMA.

- Weigh opportunities and barriers to institutionalizing a government-led total market approach.

- Identify evidence gaps and how best to address them.

- Understand whether there are resources (donor and government) available to support increased cross-sector coordination.

- Recommend first steps toward the development of a TMA plan..

\section{TMA}

\section{STAKEHOLDERS}

Government actors in the

health sector and other

sectors, such as finance,

planning, drug, and phar-

maceutical regulatory

agencies.

Development partners,

such as United Nations

agencies; bilateral agen-

cies; nongovernmental

agencies focused on FP;

and social marketing or-

ganizations, NGOs that

provide family planning

services, civil society, and

faith-based organizations.

For-profit commercial

sector agents, such as pri-

vate practitioners, clinics,

hospitals, pharmaceutical

importers, wholesalers,

distributors, and manufac-

turers. These may include

professional associations,

networks of distributors,

large private health facili-

ties or medical institutions,

health care franchises,

private insurers, and

in-country manufacturers

of FP products. 


\section{Activity 2.3. Assemble and prioritize a stakeholder list}

Assembling a truly relevant, comprehensive list of stakeholders can be labor-intensive but is well worth the effort.

\section{Developing a long list of stakeholders}

The desk review and preliminary meetings are useful to identify stakeholders. Reports and other documents collected during the desk review highlight organizations active in the FP market and the specific focus of their work. Stakeholders may be identified at the organizational and individual levels. Some individuals have perspectives and insights that are different from that of the organizations they represent (perhaps they recently joined an organization or had a unique experience within or outside that organization). It is also likely that stakeholders at the global level have relevant experience in the country and are willing to be interviewed.

In many contexts, the commercial sector is constantly evolving and includes a disparate group of stakeholders, making it harder to engage overall. This group may not see the benefit of participating in what they perceive to be a time-consuming and possibly fruitless process. Nevertheless, perspectives from actors in the commercial sector are critical. It is important to make a concerted effort to seek out their experiences and understand their perspectives in this early stage. One place to start is reviewing the membership of forums such as sector-wide associations, reproductive or maternal and child health technical working groups, or contraceptive security working groups. For the commercial sector, TMA planners can contact professional associations, looking at lists of manufacturers, large distributors, and importers who are registered to bring in and sell contraceptives.

Additional stakeholders may include representatives from the commercial sector who are already actively involved in established multisectoral forums focused on FP. It can also be useful to research organizations' websites. Finally, the acknowledgment sections of reports and studies identified in the desk review can offer a picture of how different communities of stakeholders come together to support common areas of concern and can provide additional names of people or groups to interview.

In some settings, and depending on resources and specific interests, it may be useful to develop stakeholder network maps. A visual representation of networks and stakeholders can provide an easily interpretable image of connections between actors and organizations and inform strategies and plans. Network mapping is useful to identify key stakeholders, understand the frequency and purpose of their interactions, and appraise their relative power. There is no one correct way to map stakeholder and network interactions. Tool 5 (Appendix 1) provides sample network maps and instructions.

\section{Developing a short list of key informants}

Once a full list of stakeholders has been gathered, TMA planners may also need to develop a short list of individuals to interview based on time, resources, and goals.

When shortlisting, bear in mind that some of the individuals who were contacted during the stakeholder scoping with key informants may need to be contacted again. They may want to know why they are being interviewed by the same people on the same topic twice. In these cases, planners can explain that the first key informant interview was much more general and the second interview is an opportunity to engage in more specific topics.

The objectives for stakeholder analysis developed in Step 1 help to determine who to interview. The more diverse and nuanced an issue, the more likely one will encounter varying perspectives that require broader stakeholder input

\section{Tools for Activity 2.3}

Tool 5: Sample Network Maps 
In this activity, TMA planners should develop or adapt a data collection instrument, or set of instruments, to capture key informant or stakeholder information and perspectives. The issues identified in the preliminary consultations and desk review help determine the topics these instruments should explore. It is important to keep in mind that all interview tools need to be consistent with the type of analysis to be carried out.

The focus of these tools is formed by key questions of interest, as defined by stakeholder analysis objectives (Activity 2.2) and other preliminary landscape assessment activities. For example, data may focus on identifying substantive gaps in the market and the cause of those gaps, possible solutions to overcome gaps, or what it would require (from a programmatic perspective) to implement those solutions.

The sample stakeholder interview guide in Tool 4 (Appendix 1) can be adapted to help gather information about topics not fully addressed in the desk review.

TMA planners may need to develop different versions of the instrument for different stakeholder groups. Questions for public- and private-sector informants may overlap, but it is also important to ask targeted questions of different sectors in appropriate and nuanced ways. It is important to factor in sufficient time to explain the term TMA and its relevance to the specific situation.

It may be helpful to develop a short and long version of the interview guide. A shorter version that touches on the most critical questions can be used when an interviewee indicates limited time available for the interview. The key is to prioritize the most critical questions based on what was learned during Step 1.

In determining the best approach to data collection, consider a combination of both quantitative and qualitative questions. Quantitative responses, such as supply chain indicators, delivery statistics, and quantities sold, provide an opportunity to confirm specific information gleaned during the desk review, whereas qualitative responses can add nuance and richness to the analysis.

It is recommended that TMA planners select an interviewer who is comfortable enough with the TMA to ask clarifying and probing questions as needed, as well as contribute to or lead the analysis of findings. Take the time needed to pretest the interview guide for timing as well as for redundancy and clarity of questions. Depending on the country, data collection instruments may also need to be translated and then tested in translation. Finally, in situations where resources are limited it may be advisable to convert the interview guide into an online survey instrument. An online survey will not provide the depth of information an interview can and does not allow opportunity for probing questions. However, it can provide useful insights if resources are limited. There are several free programs that can be found using any Internet search engine.

\section{Tools for Activity 2.4}

Tool 4: Guidance on Research Determination Process, Sample Research Protocol on TMA Landscaping, Sample Informed Consent Form, and Sample Generic Stakeholder Interview Guide. 


\section{Activity 2.5. Interview stakeholders}

Successfully interviewing stakeholders in a way that produces high-quality information begins with basic, but crucial, planning:

- Set up a formal schedule well in advance of carrying out interviews.

- Interview a broad range of stakeholders representing the different sectors likely to be involved in the TMA process, or who could potentially be affected by it.

- Generally conduct a minimum of 20 interviews, though the exact number will depend on the size and complexity of the overall FP environment.

- With as much lead time as possible, send all stakeholders a letter or email defining TMA and its role in the specific context, outlining the purpose of the landscape assessment, and suggesting a date/time for the interview.

- Plan sufficient time to conduct interviews in a relaxed, unhurried manner-40 minutes to an hour is generally sufficient. It is important to consider this when developing the interview guide. As mentioned above, there may be instances when the interviewee is available for a more limited time. In this case, use the short version of the interview guide.

- If required by the IRB, ask stakeholders to sign an informed consent form prior to the interview. Whether a written form is needed depends on the specific protocol and IRB approvals mentioned in Activity 2.1. This may create challenges when interviewing private-sector informants, and a result an IRB exemption may be required.

- When developing the interview schedule, leave some time slots open because informants usually suggest other people to interview.

The following tips are useful to ensure a productive interview:

- Creating a relaxed environment contributes to a rich interview experience. It is important for interviewers to practice—and convey—neutrality and to avoid bias.

- At the outset, clearly state the objectives of this inquiry and how the information will be used.

- As part of the informed consent process, the interviewer needs to clearly articulate measures that are in place to ensure that the interview content remains confidential. This is critical because controversial perspectives on the FP environment may be discussed.

- It can be helpful if two people attend, so that one person can facilitate the interview and one person can take notes. If appropriate and acceptable to the stakeholder, the interview can also be recorded. ${ }^{2}$

\section{Tools for Activity 2.5}

Tool 4. Guidance on Research Determination Process, Sample Research Protocol on TMA Landscaping, Sample Informed Consent Form, and Sample Generic Stakeholder Interview Guide.

2 For more information about best practices for interviews, please refer to: Leech BL. Asking questions: Techniques for semi-structured interviews. Political Science and Politics. 2002;35(4): 665-668. Available at: https://itp.nyu.edu/classes/interspecies/interview_technique/AskingQuestions.pdf 


\section{Activity 2.6. Update desk review}

This activity is an opportunity to revisit the desk review conducted in Step 1 and incorporate additional resources. TMA practitioners may come across helpful resources that they wish to add to the desk review throughout the various steps in the landscape assessment process, such as the stakeholder interviews, informal market check, or through their own ongoing research. It is important to revise the desk review to represent information gathered during the stakeholder interviews in order to support the analysis process and facilitate future work. After speaking with the key stakeholders during the interview process, planners should have gathered a wider range of TMA-relevant documents, such as policy or other recently approved government strategy documents, project evaluations, presentations providing details on upcoming FP projects, published survey reports, and other gray literature. A comprehensive collection and analysis of all existing FP data sources needs to done before recommending potential future market research..

The following questions may be helpful to keep in mind throughout the informal market check:

- What types of FP commodities are available and how many are generally kept in stock?

- If FP commodities are available, where are they procured from and how secure is the source?

- If FP services are available through this location, what are the costs?

- What do the commodities cost to procure and what is the final sales price to the public? Are subsidized or sliding scale options available to consumers?

- What is the client base like? Which market segments do they represent? 


\section{STEP 3. ANALYZE, VALIDATE, AND PLAN FOR NEXT STEPS}

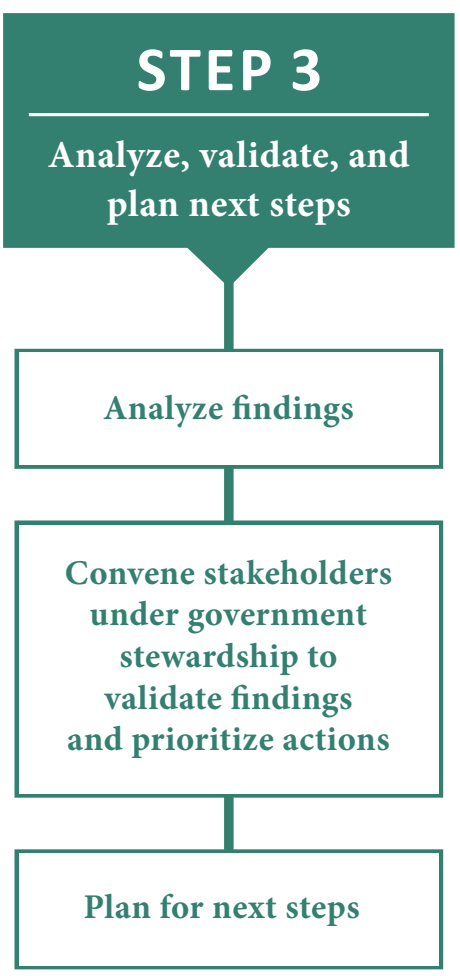

\section{WHY THIS STEP IS IMPORTANT}

Once the desk review and stakeholder interviews are complete, it is time to analyze the findings and interpret the results. Another part of this step is convening stakeholders to share findings, validate results, and identify priorities for future work with a focus on those who will benefit from TMA programming.

Analysis allows TMA planners to identify salient themes; identify key influencers, country readiness, and potential challenges; and make recommendations for implementation. Sharing results with stakeholders provides a valuable opportunity for them to discuss and validate the accuracy and relevance of the findings. It is also a chance to establish a set of recommended policy or programmatic options that address stakeholders' concerns.

Overall, Step 3 facilitates buy-in from stakeholders for a shared agenda. It is a clear transition point in the TMA process-from the landscape assessment to more action-oriented planning and secondary data analysis that will be further described in Modules 2 and 3.

\section{WHAT TMA PLANNERS SHOULD KNOW}

Step 3 is the culmination of the landscape assessment and a critical opportunity to set the stage for total market strategies in the future. Many of the stakeholders have participated in interviews and are eager to hear findings of the inquiry and participate in follow-up discussions and planning. The ministry of health counterpart, the primary FP coordination body, and TMA champions are generally considered high-priority stakeholders and have expressed an interest in working together. This provides another good opportunity to articulate and demonstrate the government's critical and evolving stewardship role, as noted in Step 1. It is also important to recognize that additional resources need to be made available and earmarked to support technical assistance and capacity-building for governments during these early stages in order for them to play a productive stewardship role.

\section{ACTIVITIES}

\section{Activity 3.1. Analyze findings}

TMA planners can use a variety of approaches to analyze the findings of the stakeholder interviews, such as qualitative computer programs MAXQDA and ATLAS.ti.

Using data obtained from the desk review and stakeholder interviews, TMA planners can use a radar map to identify the actors and organizations that exist in a country or area, as well as the strength of the connections between them. This tool 
is created by taking quantitative data and using a computer spreadsheet (such as Microsoft Excel) to compute the data and create a visual representation (see Appendix 1, Tool 5.3, for detailed instructions). It uses responses to specific questions that ask respondents to quantitatively rank the levels of influence, awareness, and support of various stakeholders in their country. In some instances this extra effort to weigh data in proportion to the power or influence of particular stakeholders interviewed can be helpful. Policy network mapping is another tool that helps to identify policies, actors, and relationships that may support or hinder future TMA programming. Both the star or radar diagrams and policy network mapping provide visual representations that can be used to illustrate these connections.

By using various methods outlined in Appendix 1 (such as Tools 4.4, 5.2, and 5.3), TMA planners can compare the analysis with interview notes or transcripts to identify themes that resonate among individuals or types of respondents (such as donors, government, or NGO staff) or that were distinct to a particular individual. Generally, information coding follows an inductive approach, with codes and themes evolving organically from the meeting notes.

This overall analysis of findings will also allow TMA planners to explore convergence (or divergence) among stakeholders' views. It will also be possible to gauge the potential success of a TMA initiative in terms of achieving intended results, implementation processes, unexpected constraints, anticipated new developments/projects, and recommendations for future TMA work.

The analysis can be further distilled by:

1. Identifying and documenting key data and evidence gaps. These may be observed when carrying out the qualitative analysis of the stakeholder interviews or during the desk review. Examples of common evidence gaps found during the landscape assessment process include:

- Overall market size, broken out by public, social marketing/NGO, and commercial sectors.

- Consumer profile data on key population groups (e.g., poor, rural, young).

- Strategies for the distribution of free or subsidized products to reach specific user groups, which ensures access to products among vulnerable populations.

- Studies that highlight how donated FP products are delivered to the same populations using multiple supply chains.

2. Applying a set of key country readiness considerations. This serves as a useful aid in the systematic qualitative analysis of stakeholder interviews. The broad questions in Tool 2 (Appendix 1) can be used as a basis for identifying and coding recurrent themes during the qualitative analysis. They can correspond to key themes from the data collection instruments and allow the team to filter and categorize the most relevant data and to help evaluate whether taking the next TMA step (outlined in Module 2) is prudent.

3. Developing a strengths, weaknesses, opportunities, and threats (SWOT) analysis matrix to consolidate and organize the data. By dividing data into the SWOT categories, planners can more clearly and effectively highlight and evaluate whether to move forward and identify what specific opportunities exist and the logical next steps in a specific country context and environment.

\section{Tools for Activity 3.1}

Tool 2: Considerations When Evaluating TMA-Related Work in Different Country Contexts

Tool 5: Sample Network Maps 
It is critical to share findings from the desk review and stakeholder interviews with those who contributed to them and others who may have an interest. A dissemination meeting is the best forum because it allows for exchange and brainstorming among participants. It is also a good opportunity for stakeholders to come together to validate the information gathered and identify next steps. As TMA planners share findings, they should be sure to present material, trends, or themes in a neutral and unbiased fashion. It is important for the stakeholders to note that data may be incomplete or nonexistent. While it is important to acknowledge these gaps, ultimately it may be necessary to make certain decisions without having the full picture.

For some stakeholders, particularly those from the commercial sector, the dissemination meeting may be the first opportunity they have to engage in discussions with government and other stakeholders regarding the FP commodity and service markets. To persuade for-profit and commercial-sector actors to attend, it may be useful to explain how engagement could work in their interest and bring long-term benefits. Acquiring meaningful commercial-sector input for a national total market strategy can be difficult. Many donors and implementing partners have little experience working with commercial actors and tend to leave them out of the discussion about total market approaches. Many companies do not see the financial benefits of participating in these forums.

\section{The role of the government}

Government stewardship of this data validation process is important to convey and ensure its importance and legitimacy. Ideally, an appropriate government representative should convene or co-convene the dissemination meeting and moderate key sessions. It is also best if the government representative is prepared to articulate a commitment to clear action moving forward and specific mechanisms for ongoing stakeholder engagement. Planners should share key informant interview key findings with government partners before convening the larger group. This provides them with an opportunity to express any reservations about what is being proposed. If their response is favorable, key messages can be developed jointly in terms that resonate with the larger group. Bear in mind that in many contexts, governments will need supplemental financial and technical support in order to carry out this vital stewardship role.

The TMA champion or team can also play an important role in supporting and preparing the government to lead this meeting. For example, it may be useful to organize a separate meeting with government to share draft findings from the stakeholder interviews and discuss possible strategies moving forward. In the process of planning the timeline for this meeting, it is also useful to allocate time for ministries of health to seek endorsement and participation from other ministries (e.g., planning and finance).

\section{Other materials to present}

In addition to these findings, the dissemination meeting may provide a good platform to present relevant government plans and strategies, updates from the private sector, information on other relevant interventions, and reports from key stakeholders. In doing so, the emphasis should be on demonstrating how these other interventions may align or conflict with the findings of the landscape assessment.

The meeting is another opportunity to reiterate the value and purpose of a TMA by highlighting examples of specific issues it could address. For example, it might be useful to explain that a TMA can reduce unmet demand for FP by correcting previously uncoordinated health outreach efforts that have left some areas of the country with low demand facing an overabundance of supplies and service providers. 


\section{Participation}

It is important to ensure that all participants in the dissemination meeting can-and do-engage and contribute. One useful method is to convene small discussion groups, in which participants reflect on, discuss, and validate the findings of the stakeholder interviews. To facilitate these small discussion groups, it may be a good idea to come up with structured topics and questions for each group. Each breakout group can then summarize their findings and recommendations to the plenary group during the final wrap-up session.

Overall, the dissemination meeting is a critical opportunity for stakeholders to articulate a shared vision of next steps for total market strategies and to establish mechanisms that support coordinated action over time.

\section{Tools for Activity 3.2}

Tool 6: Sample Agenda for Stakeholder Dissemination Meeting and Sample Outline for Breakout Groups Tool 7: How Participating in TMA Work Can Benefit the Commercial Contraceptive Sector-Pitch Sheet

Activity 3.3. Plan for next steps

At the close of the dissemination meeting, there will likely be significant momentum and a sense of purpose among participants. Many will feel it is time to move to the next steps of TMA planning. Ideas for activities and goals to pursue, and how to pursue them, will be fresh, and government leaders should have a clear perspective on the expectations of various stakeholders. Furthermore, gaps in knowledge of the contraceptive marketplace will be clearly identified. This is an appropriate time to ask who might be in a position to fund the next steps in the TMA process. It should be possible to take advantage of this momentum to initiate discussions with the government and, potentially, a core group of stakeholders about planning for a TMA. Planning may include:

- Developing a formal agreement (e.g., a memorandum of understanding or project agreement) with the government to ensure rigorous implementation of total market stewardship

- Developing or adapting terms of reference for a coordination group that clearly defines roles and responsibilities.

- Planning a more in-depth landscape assessment of the contraceptive market through market segmentation analysis (Module 2).

- Conducting a more comprehensive assessment of government stewardship capacity (Module 2).

It is also possible that the landscape assessment process will reveal little need for a concerted TMA, or that more information is required to determine whether to move forward, or that the timing is simply not right. Any of these outcomes should be considered valuable lessons.

Follow-up from the dissemination meeting should include time to talk with government and other key stakeholders about whether there is sufficient interest and support to move a TMA forward. The answer will depend on the needs identified, directions suggested by available data, and recommendations from the dissemination meeting. It is also important to solidify mechanisms for communication with and between the meeting participants going forward. 


\section{CONCLUSION}

By leveraging the comparative advantages of the public and private sectors, a total market approach can be an effective means to enhance equitable and sustainable access to high-priority FP products and services. A TMA provides a flexible framework that combines key market data with strengthened multisectoral coordination mechanisms to better meet the FP needs of all segments of a population. Effective coordination and engagement across sectors does not happen organically and requires well informed deliberation.

A landscape assessment plays a pivotal role in identifying essential background information, engaging stakeholders, and developing strategies for increased stakeholder collaboration. The activities and tools presented in this document and appendix will help TMA planners to collect the research, identify the stakeholders, and better define whether there are opportunities to pursue the next steps in the TMA process.

This landscape assessment module is the first of two modules that outline the steps needed to conduct an in-depth landscape assessment and market segmentation analysis of the FP market environment in a particular country. There are plans to develop two additional modules that focus on the subsequent steps of developing and implementing a TMA plan. As this work progresses in different country settings, the various processes outlined in this guide will be updated and revised to ensure consistency with best practices and incorporate new knowledge from ongoing FP work in the field.. 


\section{REFERENCES}

1. Barnes J, Vail J, Crosby D. Total Market Initiatives for Reproductive Health. Bethesda, MD: Strengthening Health Outcomes through the Private Sector Project, Abt Associates; 2012. Available at: http://www.popline.org/node/562153.

2. Total Market Approach page. Global Health Learning Center website. Available at: http://www.globalhealthlearning.org/course/total-market-approach.

3. Rao R, Olson N, Bornbusch A (ed.), Pilz K (ed.). The SPARHCS Process Guide: A Planning Resource to Improve Reproductive Health Commodity Security. Baltimore, MD: Information and Knowledge for Optimal Health (INFO) Project/ Center for Communication Programs, Johns Hopkins Bloomberg School of Public Health; 2008. Available at: http:// www.rhsupplies.org/fileadmin/uploads/rhsc/Tools/SPARHCS/Documents/SPARHCSProcessGuide EngPDF 1 .pdf.

4. Barnes J, Armand F, Callahan S, Revuz C. Using Total Market Approaches in Condom Programs. Bethesda, MD: Strengthening Health Outcomes through the Private Sector Project, Abt Associates; 2015. Available at: http://www.abtassociates.com/reports/2015/using-total-market-approaches-in-condom-programs.aspx.

\section{APPENDIX 1. TOOLS AND RESOURCES}

Tool 1: $\quad$ Documents for General Landscaping Desk Review

Tool 2: $\quad$ Considerations When Evaluating TMA-Related Work in Different Country Contexts

Tool 3: $\quad$ Generic TMA Overview—Presentation

Tool 4: $\quad$ Guidance on the Research Determination Process, Sample Research Protocol on TMA Landscaping, Sample Informed Consent Form, and Sample Generic Stakeholder Interview Guide

Tool 5: $\quad$ Sample Network Maps

Tool 6: $\quad$ Sample Agenda for Stakeholder Dissemination Meeting and Sample Outline For Breakout Groups

Tool 7: $\quad$ How Participating in TMA Work Can Benefit the Commercial Contraceptive Sector- Pitch Sheet 


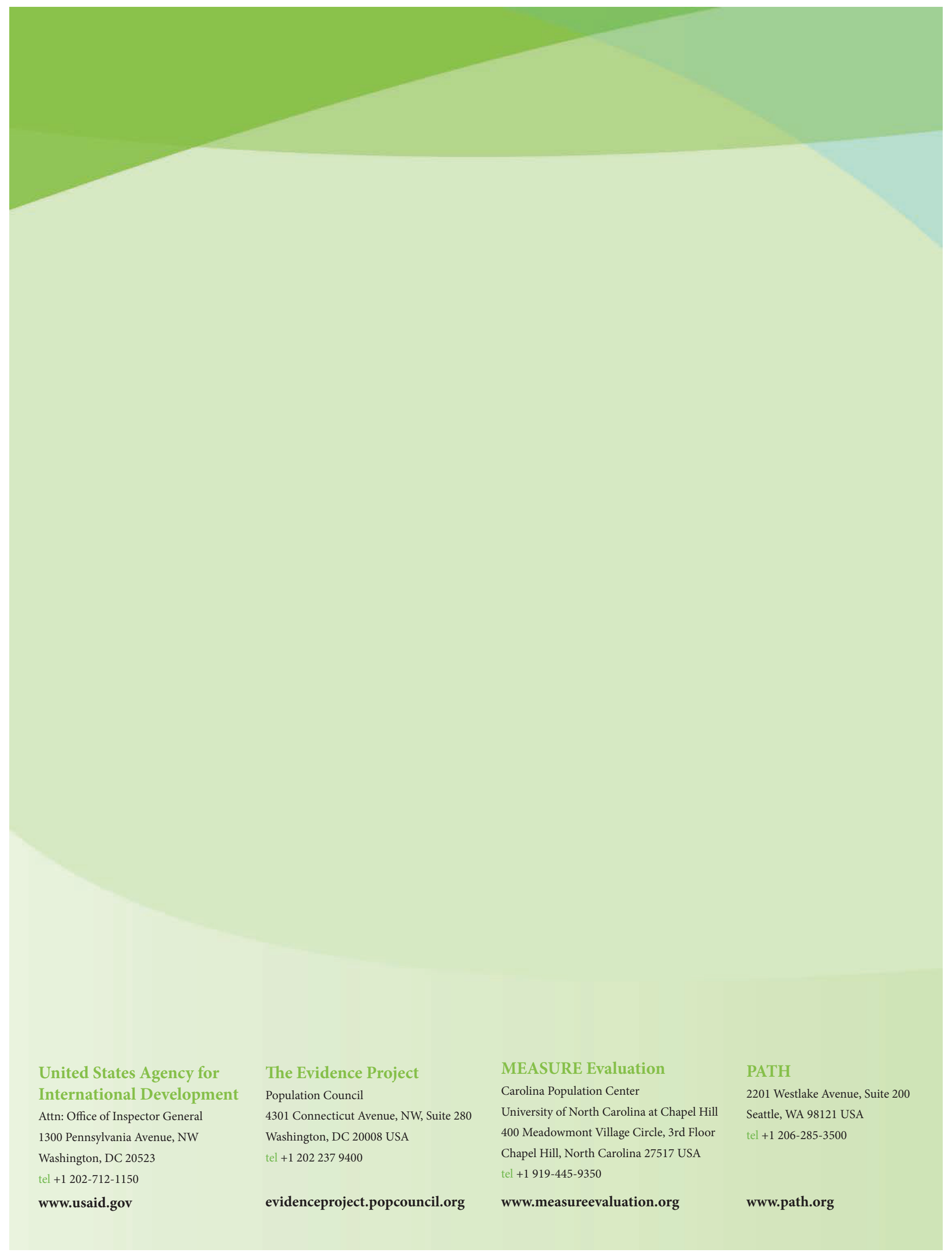

